\title{
Laparoscopia Diagnóstica en Ginecología
}

\author{
Dres.: Nelson Lenis Nicholls*, Jesús Herney Cifuentes Perea*, \\ Mario Calle Mesa** y Carlos A. Esparza Duque*** \\ HOSPITAL UNIVERSITARIO DE CALDAS \\ DEPARTAMENTO DE GINECO-OBSTETRICIA
}

\section{INTRODUCCION}

La Laparoscopia permite la exploración de la cavidad abdominal y pélvica, mediante una técnica rápida, sencilla, de bajo riesgo y de gran utilidad. Su historia se remonta al descubrimiento y desarrollo de instrumentos ópticos y de iluminaciones, el siglo pasado (18).

Se empezó a utilizar en nuestro medio a principios de la década del 70 con fines terapéuticos (electrocoagulación tubaria), en los programas de planificación familiar, y casi simultáneamente, con fines diagnósticos, en algunos hospitales universitarios.

Nuestro Departamento de Ginecología y Obstetricia* inició en 1975 el Programa de Laparoscopia con propósitos

\footnotetext{
* Médico Residente Tercer Año. Autores.

** Jefe del Servicio de Ginecología, Obstetricia. Coautor.

*** Profesor Asociado Ginecología, Obstetricia. Coautor.

* Facultad de Medicina, Universidad de Caldas. Manizales.
}

diagnósticos y terapéuticos; desde un comienzo fueron registrados los casos atendidos, de las dos actividades, en formularios precodificados que facilitaran el control y procesamiento de los datos.

\section{Material y Métodos}

El Programa de Laparoscopia, desde su inicio en enero de 1975, hasta junio de 1981, realizó un total de 1.606 procedimientos, de los cuales 1.138 correspondieron a esterilizaciones tubarias que son objeto de otro estudio; las laparoscopias con fines diagnósticos fueron 468 , que representan el $29.1 \%$ del total; de éstas se seleccionaron 415 que correspondían al período de enero 1976 a junio de 1981 y estaban registradas en formularios especiales; fueron eliminados 53 casos por presentar datos incompletos.

Las mujeres a quienes se les practicó laparoscopia con fines diagnósticos, fueron sometidas previamente a un examen clínico general y ginecológico, se les hizo historia clínica y se solicitaron los exámenes paraclínicos necesarios, además de la evaluación preanestésica. La laparoscopia fue electiva en su mayoría, 
motivo por el cual el procedimiento se hizo ambulatorio, citando a la paciente una hora antes y dándole de alta una vez recuperada de la anestesia. Sólo permanecieron hospitalizadas las pacientes ya internadas por otras causas, para quienes la laparoscopia fue indicada como ayuda diagnóstica.

Las principales indicaciones diagnósticas fueron:

\section{Estudio de esterilidad}

Dentro del esquema seguido por el Departamento de Ginecología y Obstetricia en el estudio de esterilidad, se realiza historia clínica completa, prueba de Sims-Huhner y otros. Si hay indicación para hacer la laparoscopia, ésta se realiza en la época ovulatoria o premestrual, con el objeto de observar ovulación, patología tubaria, ovárica o pélvica. Se hace a la vez biopsia de endometrio y cromopertubación. Esta secuencia es seguida por varios autores $(3,4,5,6$,$) .$

\section{Indicación diagnóstica}

La laparoscopia como procedimiento diagnóstico fue utilizada para confirmar o ayudar en casos de duda, en procesos patológicos pélvico-abdominales, masas palpables, amenorrea primaria o secundaria, dolor pélvico, perforación uterina, etc. procedimiento que se ha extendido ampliamente como auxiliar diagnóstico $(1,2,9,10,11,17,19)$.

Fueron utilizados equipos convencionales de fuentes de luz, telescopio, pinzas, agujas de insuflación de gas, trócares, así como equipo de biopsia de endometrio y cánulas de Semm para cromopertubación $(1,2,4,8,19)$. La técnica y el instrumental no se describen en detalle por no ser objetivo del estudio. De acuerdo a las necesidades, el procedimiento fue complementado con técnicas de electrocoagulación, biopsia de endo- metrio, movilización instrumental del útero, cromatoscopia para visualizar permeabilidad tubaria, liberación de adherencias laxas, etc.

Los datos obtenidos fueron registrados en formularios precodificados, cuyas características de diseño son similares al modelo del Programa Internacional de Investigaciones en Fecundidad (PRIF). Se adicionaron 21 códigos que registran los diagnósticos más importantes. La información fue procesada en la Corporación Centro Regional de Población (CCRP).

\section{Resultados}

Se presentan algunos de los resultados más importantes del estudio.

Tabla No. 1

\section{DISTRIBUCION POR EDADES}

\begin{tabular}{|crc|}
\hline Grupos de edad & Número & Porcentaje \\
\hline 19 o menos & 49 & 11.9 \\
$20-29$ & 215 & 52.6 \\
$30-39$ & 126 & 30.8 \\
$40-49$ & 15 & 3.7 \\
50 ó más & 4 & 1.0 \\
\hline Total: & 409 & 100.0 \\
\hline
\end{tabular}

Promedio: 27.4

Los extremos límites de edad fueron de 13 y 64 años, con un promedio de 27.4. Las mujeres en su mayoría (215), correspondian al grupo de edad 20 a 29 años, con el 52.6. El segundo grupo de edad fueron las mujeres entre 30 y 39 años, seguidas de las pacientes menores de 19 años. Tabla No. 1

Las mujeres procedían en su mayoría de Manizales (88.6), casi todas del sector 
urbano (73.7). El 66.6 eran mujeres casadas. Tablas Nos. 2 y 3.

Tabla No. 2

\begin{tabular}{|lrc|}
\hline Residencia & Número & Porcentaje \\
\hline Urbana & 302 & 73.7 \\
Rural & 61 & 14.9 \\
Urbana fuera del área & 24 & 5.8 \\
Rural fuera del área & 23 & 5.6 \\
\hline Total: & 410 & 100.0 \\
\hline
\end{tabular}

Tabla No. 3

\begin{tabular}{|lrr|}
\hline Estado Civil & Número & Porcentaje \\
\hline Soltera & 130 & 31.7 \\
Casada & 273 & 66.6 \\
Unión libre & 5 & 1.2 \\
Viuda & 2 & 0.5 \\
\hline Total: & 410 & 100.0 \\
\hline
\end{tabular}

El nivel de instrucción de estas mujeres fue relativamente bajo, pues sólo el 14.2 habian cursado la secundaria completa y el 18.3 habian llegado a la enseñanza superior, muchas de ellas sin completarla. Tabla No. 4.

El promedio de peso fue de 53.9 kilogramos, con un rango de 32 a 86 kilos de peso.

La fecundidad, medida por el número de nacidos vivos tenidos por cada mujer, se puede considerar baja en este estudio. El 57.8 de las mujeres (240) no habian tenido hijos; a este grupo le sigue las mujeres que sólo habian tenido de 1 a 2 hijos en el 26.3 del total.
Tabla No. 4

\begin{tabular}{|lcc|}
\hline Escolaridad & Número & Porcentaje \\
\hline Analfabeta & 10 & 2.4 \\
$\begin{array}{l}\text { Primaria } \\
\text { incompleta }\end{array}$ & 78 & 18.8 \\
$\begin{array}{l}\text { Primaria } \\
\text { completa }\end{array}$ & 53 & 12.8 \\
$\begin{array}{l}\text { Secundaria } \\
\text { incompletə }\end{array}$ & 139 & 33.5 \\
$\begin{array}{l}\text { Secundaria } \\
\text { completa }\end{array}$ & 59 & 14.2 \\
Superior & 76 & 18.3 \\
\hline Total: & 415 & 100.0 \\
\hline
\end{tabular}

Promedio: 7.8

Tabla No. 5

HIJOS NACIDOS VIVOS

\begin{tabular}{|lrc|}
\hline & Número & porcentaje \\
\hline Ninguno & 240 & 57.8 \\
$1-2$ & 109 & 26.3 \\
$3-6$ & 45 & 10.8 \\
7 o más & 21 & 5.1 \\
Total: & 415 & 100.0 \\
\hline \multicolumn{2}{l}{ Promedio 1.1} & \\
\hline
\end{tabular}

En cuanto a la forma de terminación del último embarazo, el 51.0 de lasmujeres no habian tenido embarazos $y$ un 14.3 de éstos habían terminado en aborto, mortinato o embarazo ectópico.

El uso de métodos de planificación familiar fue muy bajo, el 73.1 no utilizaba ningún método. 
Tabla No. 6

TERMINACION DEL ULTIMO EMBARAZO

\begin{tabular}{|lrr|}
\hline & Número & Porcentaje \\
\hline Sin embarazo & 207 & 51.0 \\
Nacido vivo & 141 & 34.7 \\
Mortinato & 5 & 1.2 \\
Aborto & 47 & 11.6 \\
Embarazo ectópico & 6 & 1.5 \\
\hline Total & 406 & 100.0 \\
\hline
\end{tabular}

Tabla No. 7

ANTICONCEPCION PREVIA

\begin{tabular}{|lrc|}
\hline \multicolumn{1}{|c}{ Método } & Número & Porcentaje \\
\hline Ninguno & 297 & 73.1 \\
DIU & 39 & 9.6 \\
Orales & 34 & 8.4 \\
Esterilización & 2 & 0.5 \\
Otros & 34 & 8.4 \\
\hline Total & 406 & 100.0 \\
\hline
\end{tabular}

De las mujeres con antecedentes quirúrgicos, 33 habían sido sometidas a cirugía abdominal y 39 a algún tipo de cirugía pélvica.

En el examen ginecológico que se realizó, previo a la laparoscopia, se hizo diagnóstico de miomatosis en 10 casos $y$ de tumor pélvico de origen anexial, en 23 mujeres.

Las complicaciones relacionadas con el procedimiento laparoscópico fueron pocas, menos del $2.0 \%$ requirieron hospitalización durante unas 24 horas. Estas consistieron principalmente en laceración del cérvix, perforación uterina con el elevador o hemorragia de poca consideración.

El procedimiento fue realizado con anestesia general, en el 88.0 de las mujeres. Para el resto (12.0) fue empleada la técnica de analgesia y anestesia local.

\section{Hallazgos Laparoscópicos}

Los diagnósticos laparoscópicos se distribuyeron en 4 grupos, por entidades clínicas afines, para facilitar su presentación. La suma de los diagnósticos es superior al número de mujeres, ya que algunas presentaban dos o más entidades patológicas.

Tabla No. 8

HALLAZGOS LAPAROSCOPICOS -GRUPO I-

\begin{tabular}{|lrc|}
\hline Diagnóstico & Número & Porcentaje \\
\hline E.P.I. Crónica* & 99 & 23.9 \\
Endometriosis & 52 & 12.5 \\
S. Adherencial & 47 & 11.3 \\
E.P.I. Aguda & 7 & 1.7 \\
Fitz-Hug-Curtis & 3 & 0.7 \\
Peritonitis & 1 & 0.2 \\
\hline Sub-total & 209 & 50.3 \\
\hline
\end{tabular}

(*) Incluye :Hidrosalpinx-TBC (5)

En 86 pacientes (20.7) la laparoscopia fue reportada como normal. Muchas de estas pacientes fueron sometidas a este procedimiento por dolor pélvico.

En el grupo I la enfermedad pélvica inflamatoria (aguda y crónica), ocupó el 
primer lugar con un 25.6. La endometriosis, el segundo lugar, con un 12.5 .

El sindrome adherencial se encontró en 47 pacientes. Cinco casos fueron considerados presuntivamente como T.B.C. genital. El síndrome de Fitz-HugCurtis o Perihepatitis Gonocóccica, fue observado en tres pacientes y la peritonitis en una que presentaba una apendicitis perforada.

En el grupo II se incluyeron las anormalidades de los genitales internos cuyos diagnósticos presuntivos se confirmaron por laparoscopia; en una proporción menor, éstos fueron hallazgos del estudio.

Tabla No. 9

HALLAZGOS LAPAROSCOPICOS -GRUPO II-

\begin{tabular}{|lcc|}
\hline Diagnóstico & Número & Porcentaje \\
\hline Poliquistosis Ovárica & 56 & 13.5 \\
Anomal ias Congénitas & 3 & 0.7 \\
S. de Rokitansky & 4 & 1.0 \\
Agenesia Uterina & 1 & 0.2 \\
S. de Turner & 3 & 0.7 \\
Disgenesia Gonadal Pura & 2 & 0.5 \\
\hline Sub-Total & 69 & 16.6 \\
\hline
\end{tabular}

La Poliquistosis ovárica constituyó el grupo mayoritario con el $13.5 \%$ (56). El sindrome de Rokitansky-Kuster-Hauser, se halló en 4 casos; el síndrome de Turner en 3; dos mujeres tenian útero didelfo y vagina doble. Ambas habían tenido parto reciente, atendido por césarea. Dos casos fueron interpretados como disgenesia gonadal pura.

El tercer grupo lo constituyen los tumores quísticos y sólidos. La miomatosis uterina se encontró en 26 pacientes (6.3). Los quistes funcionales de ovario (quistes foliculares), en un número similar al anterior. Los tumores ováricos (quistes dermoides, adenocarcinoma $y$ cistadenomas serosos) fueron extirpados y confirmados sus diagnósticos por estudio histopatológico.

Tabla No. 10

HALLAZGOS LAPAROSCOPICOS -GRUPO III-

\begin{tabular}{|lcc|}
\hline Diagnóstico & Número & Porcentaje \\
\hline Miomatosis & 26 & 6.3 \\
Quistes Funcionales & 25 & 6.1 \\
Tumores Ováricos* & 7 & 1.7 \\
Quistes del Paraovario & 5 & 1.2 \\
\hline Sub-Total & 63 & 15.3 \\
\hline
\end{tabular}

* Incluye: Q. Dermoide, Adenocarcinoma y Cistadenoma Seroso.

Tabla No. 11

HALLAZGOS LAPAROSCOPICOS -GRUPO IV-

\begin{tabular}{|lrc|}
\hline \multicolumn{1}{|c}{ Diagnóstico } & Número & Porcentaje \\
\hline Varicocele pelviano & 50 & 12.0 \\
Hipoplasia genital & 25 & 6.0 \\
Embarazo ectópico & 5 & 1.2 \\
Otros* $^{*}$ & 15 & 3.6 \\
\hline Sub-Total & 95 & 22.8 \\
\hline
\end{tabular}

* Incluye : Perforación uterina, Dispositivos extrauterinos, otros.

En el grupo IV se incluyeron, el varicocele pelviano con 50 casos (12.0), la hipoplasia de los genitales internos en 25 (6.0). Se confirmó el diagnóstico de embarazo ectópico en 5 casos de diagnóstico dudoso. En "otros" se incluyeron 15 pacientes que a la laparoscopia mostraron perforación uterina, dispositivos extrauterinos, apendicitis $y$ congestión pélvica. 


\section{Comentarios}

Se presenta un estudio de 415 casos de laparoscopia diagnóstica, realizado en el Departamento de Ginecología y Obstetricia de la Facultad de Medicina de la Universidad de Caldas, durante el período comprendido entre enero de 1976 a junio de 1981.

Este procedimiento es un extraordinario recurso que no descarta los demás métodos auxiliares de diagnóstico en ginecología, sino que los mejora $y$ complementa.

La laparoscopia se realizó como ayuda diagnóstica en el estudio de pacientes con esterilidad y para confirmar, descartar o complementar la investigación de otra patología gineco-obstétrica.

La laparoscopia ha tenido un extraordinario desarrollo en los últimos 20 años. En el presente estudio se utilizó en mujeres con edades entre los 13 y 64 años. La más joven tenía un quiste dermoide del ovario izquierdo y había sido operada cuatro años antes de un quiste similar en el ovario contralateral. La paciente de 64 años, había sido intervenida de adenocarcinoma seroso papilar bilateral de ovario, hacia 27 años, complementándose el tratamiento con radioterapia y quimioterapia. En la laparoscopia no se encontró patología intraperitoneal.

El $20.7 \%$ de las laparoscopias fueron informadas como normales, sin que se pueda inferir del estudio la indicación de cada caso por fallas en la información; muchas se realizaron como medio diagnóstico por dolor pélvico, sin encontrar patología; Amorocho y colaboradores en Cali (17), encontraron un $26 \%$ de laparoscopias negativas.

Un poco más de la mitad de las mujeres (52.9) tenían entre 20 y 29 años, grupo de edad que normalmente corres- ponde a mujeres con mayor capacidad reproductiva y que en los estudios de fecundidad realizados en el país, representan las tasas más altas (15.16). Sin embargo, en las mujeres del estudio, el 57.8 no habia tenido hijos $y$ en cuanto a la forma de terminación del último embarazo, el 51.0 eran nulíparas y en un 14.2 el embarazo había terminado en aborto, mortinato o embarazo ectópico. Estos datos se relacionan con la condición especial de estas mujeres, que en su mayoría presentaban factores de esterilidad.

Es en mujeres jóvenes que inician su vida reproductiva, en las que se encuentran las tasas más altas de uso y conocimiento de métodos anticonceptivos (7, $15,16)$. Sin embargo, en el presente estudio, el 73.2 no utilizaba ninguno. Este hallazgo estaría relacionado con la condición de esterilidad de muchas de estas mujeres.

Sin entrar a comentar en detalle todos los diagnósticos, se observa que éstos, por sus características propias como enfermedad pélvica inflamatoria, anormalidades congénitas o patología tumoral que comprometen órganos que tienen relación con la función reproductiva, determinan en primer lugar factores de esterilidad $(11,12,14)$ no analizados en este estudio; tampoco se presenta la conducta que se siguió con las pacientes, después de hacer el diagnóstico laparoscópico, porque ésto no era su propósito. El trabajo tenía como objetivo conocer los resultados de las laparoscopias $y$ analizar algunas características de las mujeres, como edad, estado civil, anticoncepción previa, paridad anterior, etc. que pudieran tener alguna relación con los diagnósticos establecidos.

Delgado y Col. $(3,4,5)$ en México, atribuye como causa de esterilidad al factor tubario en un 50.9, seguido del endocrino-ovárico con 16.4, asociado a un tipo de patología (S. adherencial, 
$E P I$, anomalías) que en nuestro estudio ocupa los principales lugares.

Este primer estudio sobre "Laparoscopia Diagnóstica" realizado en el Hospital Universitario de Caldas, habrá de servir para mejorar los procedimientos de tipo técnico y de registro de datos, definir mejor las indicaciones de su uso, intensificar su utilización y ampliar su cobertura.

\section{Resumen}

Se hace una revisión de 415 casos de laparoscopia diagnóstica, realizadas en el Departamento de Ginecología y Obstetricia de la Facultad de Medicina de la Universidad de Caldas, durante el período enero 1976 a junio de 1981.

La laparoscopia se ha utilizado como un procedimiento auxiliar para el diag- nóstico en ginecología, que sin desplazar otros procedimientos, es de gran ayuda en el estudio de la esterilidad femenina $y$ el diagnóstico de patología ginecológica.

Los datos indican que las mujeres del estudio son jóvenes en su mayoría $(52.6 \%$ entre los 20 y 29 años), con baja fecundidad, y presentan factores de esterilidad en alta proporción, según se establece por los hallazgos diagnósticos, muchos de ellos relacionados con procesos inflamatorios pélvicos (EPI, endometriosis, S. adherencial, etc.), o factores congénitos.

El estudio habrá de servir para mejorar su técnica, incrementar el registro de datos, intensificar su uso y establecer las normas más adecuadas de su utilización.

\section{DIAGNOSTIC LAPAROSCOPY IN GYNECOLOGY}

\section{Summary}

This article presents a retrospective study of 415 cases of diagnostic laparoscopy done at the Department of Obstetrics and Gynecology of University of Caldas School of Medicine during the period January 1976 - June 1981.

Laparoscopy has been used as an auxiliary diagnostic procedure in Gynecology. Without eliminating other proce-

\section{Bibliografía}

1. NAVA Y SANCHEZ R.; GARCIA MONROY L. Laparoscopia en Gineco-Obstetricia. Reporte Preliminar. Ginecología dures, laparoscopy is very helpful in the assessment of female sterility and the diagnosis of gyn pathology.

Data show that the majority of the women in this study are young $152.6 \%$ are between 20 and 29 years of age), have a high incidence of sterility factors as evidenced by diagnostic findings, and many of these were related with pelvic inflammatory processes (PID, endometriosis, etc.), or congenital factors. y Obstetricia de México. Vol. 42, julio 1977.

2. CIBILS L.A. Laparoscopia en Ginecología. Ginecología y Obstetricia de México. Vol. 37, junio 1975. 
3. DELGADO URDAPILLETA J.; RUIZ HERNANDEZ C.E.; MARRUFO RAMI-REZ J.A.; VELEZ MORENO I. La Laparoscopia como Método Diagnóstico en Ginecología. Análisis de 1.000 casos. Ginecología y Obstetricia de México. Vol. 42, Nov. 1977.

4. DELGADO URDAPILLETA J.; VILLALOBOS ROMAN M.; SANTOS GONZALEZ. J; REYES BALLIN J.; PLASCENCIA N. La Laparoscopia como Método Diagnóstico en Ginecología. Cirugía y Cirujanos, México, agosto 1974.

5. DELGADO URDAPILLETA J ; VILLALOBOS ROMAN M.; SANTOS GONZALEZ J.; REYES BALLIN J .; PLASCENCIA N. La Laparoscopia en el Estudio de la Esterilidad Femenina. Ginecología y Obstetricia de México. Vol. 37, abril 1975.

6. VILLALOBOS ROMAN M.; DELGADO URDAPILLETA J.; REYES BALLIN J. M. SANTOS GONZALEZ J . La Laparoscopia en el Estudio de la Esterilidad Femenina. Ginecología y Obstetricia de México. Vol. 35, marzo 1974.

7. CORPORACION CENTRO REGIONAL DE POBLACION. Ministerio de Salud; Westinghouse Health Systems. Encuesta Nacional de Prevalencia del uso de Anticoncepción. Colombia, 1978. Resultados Generales. Bogotá, octubre 1979.

8. MORGAN H.R.; Laparoscopy: Induction of Pneumoperitoneum via Transfundal puncture. Obstetrics and Gynecology. Vol. 59, No. 2. august. 1979.

9. KLEINHAUS S.; HEINK.; SHERAN M; BOLEY S. Laparoscopy for Diagnosis and tratment of Abdominal Pain in Adolescent Girls. Arch. Surg. Vol. 112, oct. 1977.
10. Mc. BRIDE N.; NEWMAN R. Diagnostic Laparoscopy. Int. J. Gynaecology Obstet. 15, 1978.

11. SWEET R.; COL. Use of Laparoscopy to Determine the Microbiologic Etiology of acute Salpingitis. Am. J. Obstet. Gynecol. May I, 1979.

12. PHILIPSEN T.; BALDUR B. Comparative Study of Hysterosalpingography and Laparoscopy in Infertile Patients. Acta Obstet. Gynecol. Scand 60, 1981.

13. PENFIELD A.J. Laparoscopie Sterilization under local anestesia. Am.J. Obstet. Gynecol. July 1974.

14. CASTHELY S.; MACHESWARN CH.; LEVY J. Laparoscopy: An important tool in the Diagnosis of RokitanskyK uster-Hauser Syndrome. Am. J. Obstet. Gynecol.J une. 1974.

15. CCRP. Area de Evaluación. 1978. Conocimiento de Métodos Anticonceptivos y Servicios de Planificación Familiar. Colombia, 1969 y 1976, Bogotá.

16. CCRP. DANE. ISI. 1977. Encuesta Nacional de Fecundidad, 1976. Resultados Generales. Bogotá.

17. AMOROCHO NESTOR H.; AMASTHA FARID; DE RUIZ Ma. DEL SOCORRO.

Dolor Pélvico. Revista Colombiana de Obstetricia y Ginecología. Nov. Dic., 1980; Vol. XXXI, No. 16.

18. AMOROCHO NESTOR H. Laparoscopia, Revisión de Temas, Rev. Colombiana de Obstetricia y Ginecología. Sep. Oct. 1979, Vol. XXX No. 5.

19. DAVID PENT. Laparoscopy :Its. Role iı Private Practice. Am. J. Obstet. Ginecol. June 15, 1972. 